Military Technical College Kobry El-Kobbah, Cairo, Egypt

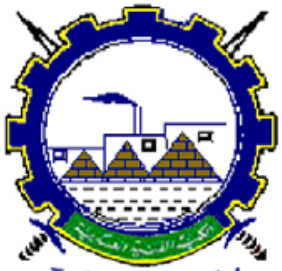

I.C.E.E.2014 $7^{\text {th }}$ International Conference

on

Chemical \& Environmental

Engineering

27 - 29 May, 2014.

AGNM-1

\title{
Graphene-Supported Metal Nanoparticles and Its Potential Civilian and Military Applications
}

\author{
Hany A. A. Elazab*

\section{Abstract}

It is now well established that nanoparticles $(1-100 \mathrm{~nm})$ exhibit unique chemical and physical properties that differ from those of the corresponding bulk materials. The dependence of the properties of nanoscale materials on the size, shape and composition of the nanocrystal is a phenomenon of both fundamental scientific interest and many practical and technological applications. These properties are often different, and sometimes superior, to those of the corresponding bulk materials.

Due to its unique properties and high surface area, Graphene has become a good candidate as an effective solid support for metal catalysts. The Nobel Prize in Physics for 2010 was awarded to Andre Geim and Konstantin Novoselov "for groundbreaking experiments regarding the two-dimensional material graphene". The graphene-supported metallic nanostructured materials were successfully used to investigate Suzuki cross-coupling reaction as an important reaction in the field of pharmaceutical applications. In recent research, nanostructured materials were used as solid support catalysts which showed remarkable improvements in the aspects of size, surface structure, catalytic selectivity, shape and recyclability. The nanoporous structure and superparamagnetic behavior of $\left(\mathrm{Fe}_{3} \mathrm{O}_{4}\right)$ nano particles that were used as an effective ingredient in graphene-supported palladium catalyst improved the catalytic activity and the catalyst recyclability simply by using an external magnetic field.

Microwave-assisted synthesis was recently used as an efficient method to prepare various metallic nanostructured materials. These metallic nanostructured materials were used to convert $\mathrm{CO}$ to $\mathrm{CO}_{2}$ as an effective approach for carbon monoxide elimination due to its harmful effect on health and environment.

The characterization of the unique properties of nanocrystals can ultimately lead to identifying many potential uses and applications, ranging from catalysis, ceramics, microelectronics, pigments, chemical and biological sensors, optoelectronics, drug delivery, and media storage. With the highly promising expectations of nanotechnology for new innovative products, materials and power sources it is evident that nanotechnology can bring many innovations into the defense world. Innovation in military technology can become an extension of warfare itself. Being ahead in technology provides an important advantage in armed conflict, and if the lead position is not attainable, then one should at least stay as close as possible behind.

\footnotetext{
Egyptian Armed Forces.
} 